Acta Haematol 2012;127:47-9.

2. Martin-Cabrera P, Haferlach C, Kern W, Schnittger S, Haferlach T. BCR-ABL1-positive and JAK2 V617F-positive clones in 23 patients with both aberrations reveal biologic and clinical importance. Br J Haematol 2017;176:135-9.

3. Park SH, Chi HS, Cho YU, et al. Two cases of myeloproliferative neoplasm with a concurrent JAK2 (V617F) mutation and BCR/ABL translocation without chronic myelogenous leukemia phenotype acquisition during hydroxyurea treatment. Ann Lab Med 2013;33:229-32.

4. Yi JH, Kim HR. Coexistence of BCR/ABL1-positive chronic myeloid leukemia and JAK2 V617F-mutated myelofibrosis successfully treated with dasatinib and ruxolitinib. Blood Res 2019; 54:77-9.

5. Bader G, Dreiling B. Concurrent JAK2-positive myeloproliferative disorder and chronic myelogenous leukemia: a novel entity? A case report with review of the literature. J Investig Med High Impact Case Rep 2019;7:2324709619832322.

6. Lewandowski K, Gniot M, Wojtaszewska M, et al. Coexistence of JAK2 or CALR mutation is a rare but clinically important event in chronic myeloid leukemia patients treated with tyrosine kinase inhibitors. Int J Lab Hematol 2018;40:366-71.

7. Agarwal R, Blombery P, McBean M, et al. Clinicopathological differences exist between CALR- and JAK2-mutated myeloproliferative neoplasms despite a similar molecular landscape: data from targeted next-generation sequencing in the diagnostic laboratory. Ann Hematol 2017;96:725-32.

8. Soderquist CR, Ewalt MD, Czuchlewski DR, et al. Myeloproliferative neoplasms with concurrent BCR-ABL1 translocation and JAK2 V617F mutation: a multi-institutional study from the bone marrow pathology group. Mod Pathol 2018;31:690-704.

9. Zhou A, Knoche EM, Engle EK, Fisher DA, Oh ST. Concomitant JAK2 V617F-positive polycythemia vera and BCR-ABL-positive chronic myelogenous leukemia treated with ruxolitinib and dasatinib. Blood Cancer J 2015;5:e351.

\section{Daratumumab in dialysis-dependent multiple myeloma}

TO THE EDITOR: Daratumumab is an IgG1 kappa monoclonal antibody against CD38, overexpressed by myeloma cells. It acts by several mechanisms, including triggering complement-dependent cytotoxicity and antibody-dependent cell-mediated cytotoxicity, antibody-dependent cellular phagocytosis, and apoptosis [1]. The combination of daratumumab, lenalidomide, and dexamethasone has demonstrated remarkable overall response rates (92.9\%) in patients with relapsed myeloma [2]. Daratumumab clinical trials have excluded patients with a creatinine clearance $<20$ $\mathrm{mL} / \mathrm{min}$ [3]. Data on daratumumab therapy in renal failure patients requiring dialysis are scarce, even though pharmacokinetic data suggest that it can be safely used without dose modification in patients with creatinine clearance $<30$ $\mathrm{mL} / \mathrm{min}$ [4]. Here, we present our experience with daratumumab in two patients with severe renal impairment.

A 36-year-old female with no previous comorbidities, presented with anemia and renal failure (serum creatinine, $8.4 \mathrm{mg} / \mathrm{dL}$; urine output, $700 \mathrm{~mL} / \mathrm{d}$ ). The $\mathrm{M}$ component was $0.22 \mathrm{~g} / \mathrm{dL}$, serum kappa $(\kappa)$ light chain was $3,650 \mathrm{mg} / \mathrm{L}$, and the lambda light chain $(\lambda)$ was $7.41 \mathrm{mg} / \mathrm{L}$. The difference in free light chain (dFLC) was 3,642 $\mathrm{mg} / \mathrm{L}$. Bone marrow evaluation demonstrated $50 \%$ clonal plasma cells. The myeloma fluorescence in-situ hybridization (FISH) panel was negative. The patient was diagnosed with IgG kappa multiple myeloma R-ISS stage III with severe renal impairment requiring dialysis and started on a cyclophosphamide, bortezomib, dexamethasone (CyBorD) regimen. After 2 weeks of CyBorD therapy, the patient developed hyperemesis due to cyclophosphamide. Cyclophosphamide was replaced with thalidomide. Bortezomib, thalidomide, and dexamethasone (VTD) were administered for 2 weeks, following which thalidomide was discontinued owing to severe myalgia. Next, the ABCD regimen, comprising liposomal doxorubicin, bortezomib, cyclophosphamide (100 mg D1-D15), and dexamethasone (40 mg weekly), was initiated. The patient tolerated the ABCD therapy well, and disease evaluation performed after two ABCD cycles presented stable disease $(\kappa>3,650 \mathrm{mg} / \mathrm{L} ; \lambda, 7.41 \mathrm{mg} / \mathrm{L}$ and $\mathrm{M}$ spike of 0.24 $\mathrm{g} / \mathrm{dL}$ ), with a continuing need for dialysis. As no disease response was observed, daratumumab $(16 \mathrm{mg} / \mathrm{kg} / \mathrm{dose}$ weekly $\times 8$ doses, followed by 2 -weekly $\times 8$ doses, then monthly), lenalidomide $(5 \mathrm{mg})$, and dexamethasone were administered. To avoid fluid overload, the daratumumab infusion was administered after the dialysis session, and the infusion rate did not exceed $100 \mathrm{~mL} / \mathrm{h}$. No infusion reactions or cytopenia were observed. The patient was dialysis-independent after the fourth daratumumab dose, reporting a serum creatinine stabilized at $4.3 \mathrm{mg} / \mathrm{dL}$. After the ninth daratumumab dose, disease evaluation demonstrated a Very Good Partial Response (VGPR) including $\mathrm{M}$ band of $0.12 \mathrm{~g} / \mathrm{dL}, \kappa-22.1$ $\mathrm{mg} / \mathrm{L}, \lambda-12.9 \mathrm{mg} / \mathrm{L}$ ( $/ / \lambda$ ratio 1.73 ), and $\mathrm{dFLC}$ of $9.2 \mathrm{mg} / \mathrm{L}$. The patient underwent an autologous stem cell transplant with high dose melphalan $\left(140 \mathrm{mg} / \mathrm{m}^{2}\right)$. Hematopoietic stem cell (HSC) mobilization was performed with the granulocyte colony-stimulating factor and upfront plerixafor. The total HSC dose collected after two sessions of apheresis was $1.52 \times 10^{6}$ cells $/ \mathrm{kg}$. Neutrophil engraftment was achieved on day 12, and platelet engraftment was achieved on day 14 . The patient was restarted on monthly daratumumab injections from day 60 post-transplant, indicating a stringent complete response $(\kappa, 2.8 \mathrm{mg} / \mathrm{L} ; \lambda, 1.8 \mathrm{mg} / \mathrm{L}$ and ratio 2.1$)$ on the day 100 evaluation, with continuing complete remission observed 21 months post-transplant on monthly daratumumab.

The second patient, a 53-year-old female, with no previous comorbidities, was diagnosed with $\lambda$ light chain myeloma RISS-III with renal failure requiring hemodialysis (serum creatinine, $7.9 \mathrm{mg} / \mathrm{dL}$; urine output, $200 \mathrm{~mL} / \mathrm{d}$ ). The 
patient demonstrated an initial serum $\lambda$ light chain of 1,620 $\mathrm{mg} / \mathrm{L}$, with a $\kappa$ light chain value of $72 \mathrm{mg} / \mathrm{L}$. Unfortunately, the myeloma FISH panel was not performed. The patient was started on bortezomib, dexamethasone, and a renal modified dose of lenalidomide for five cycles, following which disease evaluation showed complete remission. The patient was continued on maintenance bortezomib once every 2 weeks, remaining dialysis-dependent with no improvement in renal function. After 2 years, the patient relapsed while on bortezomib maintenance, with $\lambda-1,440$ $\mathrm{mg} / \mathrm{L}, \kappa-140 \mathrm{mg} / \mathrm{L}$, and a ratio of 0.097 . Next, daratumumab, lenalidomide ( $5 \mathrm{mg}$, later increased to $10 \mathrm{mg}$ ), and dexamethasone were initiated. Daratumumab $(16 \mathrm{mg} / \mathrm{kg}$ weekly $\times 8$ wk) was administered on the day after dialysis. The urine output was $400 \mathrm{~mL} /$ day. We arranged to perform dialysis post daratumumab infusion as the patient tended to retain fluid and appeared swollen. However, post-infusion dialysis was not needed. Disease evaluation performed after the eighth daratumumab dose demonstrated a partial response with $\kappa-59.7 \mathrm{mg} / \mathrm{L}, \lambda-417 \mathrm{mg} / \mathrm{L}$, and ratio of 0.143 , with a $50 \%$ reduction in dFLC. The patient developed anemia and thrombocytopenia, defaulting therapy after the eighth daratumumab dose. One year later, the patient presented with symptomatic disease and was administered daratumumab $(16 \mathrm{mg} / \mathrm{kg} /$ dose weekly $\times 8$ wk $)$ with dexamethasone and pomalidomide $(4 \mathrm{mg})$. Due to itching and diarrhea, pomalidomide was discontinued after 2 weeks. Low dose lenalidomide $(5 \mathrm{mg}$ ) was restarted but was discontinued after 2 weeks due to reported intolerance. Daratumumab and dexamethasone were continued and modified to two weekly injections after the eighth dose. Disease evaluation after the tenth dose presented stable disease, with $\kappa-56.3 \mathrm{mg} / \mathrm{L}, \lambda-233 \mathrm{mg} / \mathrm{L}$, and a ratio of 0.24 . The patient complained of new-onset back pain, and Positron Emission Tomography-Computed Tomography (PET-CT) reported multiple 18F-fluorodeoxyglucose (FDG)-avid lytic lesions. Owing to the observed disease progression, the $A B C D$ regimen was initiated. Post two ABCD cycles, the patient defaulted therapy again and was lost to follow up.

A triple combination of bortezomib, dexamethasone, and cyclophosphamide has been used to treat myeloma patients with renal failure. Lenalidomide can be prescribed in bortezomib refractory patients; dose alteration is needed in patients with a creatinine clearance $<30 \mathrm{~mL} / \mathrm{min}$. In a relapsed setting, carfilzomib and pomalidomide have demonstrated safety and efficacy in myeloma patients with dialysis-dependent renal failure $[5,6]$.

In this report, we demonstrate the safe use of combination daratumumab therapy in two patients with multiple myeloma on regular hemodialysis. One patient reported a long-lasting complete response with dialysis-independence. The other patient demonstrated a partial response to daratumumab. Nonetheless, the therapy was well-tolerated by both patients. Furthermore, one patient indicated poor mobilization of stem cells following autologous stem cell transplant, despite upfront plerixafor. In vitro studies have demonstrated that even though CD34+ stem cells indicate minimal CD38 expression, daratumumab is non-toxic to CD34+ progenitor cells in myeloma patients [7]. Poor mobilization in one of our patients could be attributed to the multiple treatments received earlier.

There are limited reports on the use of daratumumab in myeloma patients with severe renal impairment. In a Spanish retrospective multicenter trial [8], eight patients (6 with myeloma-related renal failure and 2 with pre-existing $\mathrm{CKD}$ ) were administered daratumumab in the presence of renal failure requiring dialysis. After diagnosis, the median time to daratumumab initiation was 4.6 years (range, 1-6). All patients were heavily pre-treated, with four median prior therapies. Four patients had received daratumumab after stem cell transplant (3 autologous and 1 allogeneic). At a median number of four cycles, the overall response rate was $62.5 \%$ (1 VGPR and 2 PR). However, daratumumab therapy failed to result in dialysis independence. Grade 1 or 2 infusion reactions were observed in four patients, with none discontinuing therapy due to toxicity. In a report published by Rocchi et al. [9], a 68-year-old patient, demonstrating relapsed refractory multiple myeloma post four lines of therapy, was administered single-agent daratumumab. After the ninth dose, the patient attained a stringent complete response, with no reports of infusion-related adverse events. Furthermore, the renal functions improved, necessitating a reduced dialysis frequency. Notably, our study is only the second report in which a patient demonstrated significant renal recovery post daratumumab therapy [10]. Currently, a phase II trial is evaluating daratumumab combination therapy in myeloma requiring dialysis (NCT03450 057).

There were concerns regarding the large volume of fluids to be administered with daratumumab. The manufacturer's guidelines recommended the administration of $1,000 \mathrm{~mL}$, increased to $200 \mathrm{~mL} / \mathrm{h}$ gradually. Infusion was started at $50 \mathrm{~mL} / \mathrm{h}$ and titrated as per protocol with standard dilution volumes, with no infusion-related reactions reported. The infusion rate did not exceed $100 \mathrm{~mL} / \mathrm{h}$. The second patient appeared swollen after each infusion but did not require a second dialysis. We planned daratumumab therapy immediately after dialysis to minimize the impact of fluid imbalance. In both patients, standard hemodialysis was performed using the F6 low flux dialyzer as monoclonal antibodies are not dialyzable. Now, it has been identified that daratumumab can be administered in $500 \mathrm{~mL}$ normal saline over $3-4 \mathrm{~h}$.

Our first patient demonstrated recent-onset renal failure and was dialysis-independent after therapy. The second patient had been dialysis-dependent for 3 years; hence, renal recovery was not expected. In conclusion, daratumumab can be safely administered in dialysis-dependent patients with renal failure. The effect on HSC mobilization needs to be investigated in larger studies. 
Preethi Jeyaraman ${ }^{1}$, Alka Bhasin ${ }^{2}$, Nitin Dayal ${ }^{3}$, Sangeeta Pathak ${ }^{4}$, Rahul Naithani ${ }^{1}$

${ }^{1}$ Division of Hematology and Bone Marrow Transplant, ${ }^{2}$ Department of Nephrology, ${ }^{3}$ Department of Pathology, ${ }^{4}$ Department of Transfusion Medicine, Max Super-Specialty Hospital, New Delhi, India

Correspondence to: Preethi Jeyaraman Division of Hematology and Bone Marrow Transplant, Max Super-Specialty Hospital, Saket, New Delhi 110017, India E-mail: preethijraman1@gmail.com

Received on Aug. 1, 2019; Revised on Dec. 3, 2019; Accepted on Jan. 13, 2020 https://doi.org/10.5045/br.2020.55.1.65

\section{Authors' Disclosures of Potential Conflicts of Interest}

No potential conflicts of interest relevant to this article were reported.

\section{REFERENCES}

1. Sanchez L, Wang Y, Siegel DS, Wang ML. Daratumumab: a first-in-class CD38 monoclonal antibody for the treatment of multiple myeloma. J Hematol Oncol 2016;9:51.

2. Dimopoulos MA, Oriol A, Nahi H, et al. Daratumumab, lenalidomide, and dexamethasone for multiple myeloma. $\mathrm{N}$ Engl J Med 2016;375:1319-31

3. Palumbo A, Chanan-Khan A, Weisel K, et al. Daratumumab, bortezomib, and dexamethasone for multiple myeloma. $\mathrm{N}$ Engl J Med 2016;375:754-66.

4. Yan X, Clemens PL, Puchalski T, et al. Target-mediated drug disposition of daratumumab following intravenous infusion in relapsed or refractory multiple myeloma after prior proteasome inhibitors and immunomodulatory drugs: a population pharmacokinetic analysis. Blood 2015;126:4222.

5. Dimopoulos M, Weisel K, van de Donk NWCJ, et al. Pomalidomide plus low-dose dexamethasone in patients with relapsed/refractory multiple myeloma and renal impairment: results from a phase II trial. J Clin Oncol 2018;36:2035-43.

6. Quach H, White D, Spencer A, et al. Pharmacokinetics and safety of carfilzomib in patients with relapsed multiple myeloma and end-stage renal disease (ESRD): an open-label, single-arm, phase I study. Cancer Chemother Pharmacol 2017;79:1067-76.

7. Ma X, Wong SW, Zhou P, et al. Daratumumab binds to mobilized CD34+ cells of myeloma patients in vitro without cytotoxicity or impaired progenitor cell growth. Exp Hematol Oncol 2018;7:27.

8. Cejalvo MJ, Legarda M, Abella E, et al. Activity and safety of daratumumab monotheray in patients with relapsed and refractory multiple myeloma requiring dialysis: preliminary results of Spanish, retrospective, multicenter trial. Blood (ASH Annual Meeting Abstracts) 2017;130(Suppl 1):1866.

9. Rocchi S, Tacchetti P, Pantani L, et al. Safety and efficacy of daratumumab in dialysis-dependent renal failure secondary to multiple myeloma. Haematologica 2018;103:e277-8.

10. Smyth E, Glavey S, Melotti D, et al. Dialysis independence following single-agent daratumumab in refractory myeloma with renal failure. Ir J Med Sci 2019;188:1079-80.

\section{Molecular screening for an underlying myeloproliferative neoplasm in patients with stroke: who and how?}

TO THE EDITOR: In a recent issue of Blood Research, Song and colleagues highlighted the number of patients with cerebral infarctions and either erythrocytosis or thrombocytosis in whom further investigation of a myeloproliferative neoplasm (MPN) was not sought [1]. Extrapolated globally, where the lifetime risk of stroke is approximately $25 \%$, this seemingly small number of patients with an underlying MPN would represent a considerable proportion of worldwide stroke cases in which intervention with specific MPN-directed therapies, both established and novel, would be missed [2, 3].

This important finding raises some considerations. In addition to improved communication between hematologists and neurologists, would the authors suggest implementation and justification of an MPN-associated molecular screening programme for all patients with stroke, regardless of the presence of an erythrocytosis or thrombocytosis? Furthermore, the vast majority of MPN patients presenting with stroke have molecular evidence of the JAK2 V617F mutation, however rare cases harboring $J A K 2$ exon 12, $M P L$ exon 10 and $C A L R$ exon 9 mutations have been reported [4-6]. Would the authors therefore consider incorporating these other MPN-associated driver mutations into any molecular screening programme? As MPN-directed therapy should be regarded as an integral component of secondary stroke prevention [7], identification or exclusion of this underlying malignant cause should be a priority.

Stephen E. Langabeer Cancer Molecular Diagnostics, St. James's Hospital, Dublin, Ireland

Correspondence to: Stephen E. Langabeer Cancer Molecular Diagnostics, Trinity Translational Medicine Institute, St. James's Hospital, Dublin D08 W9RT, Ireland E-mail: slangabeer@stjames.ie

Received on Feb. 17, 2020; Accepted on Mar. 7, 2020 https://doi.org/10.5045/br.2020.55.1.67

\section{Authors' Disclosures of Potential Conflicts of Interest}

No potential conflicts of interest relevant to this article were reported.

\section{REFERENCES}

1. Song IC, Choi YS, Shin JW, Song HJ, Kim J, Jo DY. Retrospective screening for Philadelphia-negative myeloproliferative neoplasms in patients with cerebral infarctions as revealed using the 